\title{
Impossibility of creating a superposition of unknown quantum states
}

\author{
Somshubhro Bandyopadhyay ${ }^{1, *}$ \\ ${ }^{1}$ Department of Physics, Bose Institute, Unified Academic Campus, \\ EN 80, Sector V, Bidhannagar, Kolkata 700091, India
}

\begin{abstract}
The superposition principle is fundamental to quantum theory. Yet a recent no-go theorem has proved that quantum theory forbids superposition of unknown quantum states, even with nonzero probability. The implications of this result, however, remain poorly understood so far. In this paper we show that the existence of a protocol that superposes two unknown pure states with nonzero probability (allowed to vary over input states) leads to the violation of other no-go theorems. In particular, such a protocol can be used to perform certain state discrimination and cloning tasks that are forbidden not only in quantum theory but in no-signaling theories as well.
\end{abstract}

In quantum theory, a state of a physical system is a vector $|\psi\rangle$ of unit norm in a Hilbert space $\mathcal{H}$. Furthermore, $|\psi\rangle$ and $e^{i \vartheta}|\psi\rangle$ describe the same physical state of the system, where $\left|e^{i \vartheta}\right|=1$; thus, a global phase is inconsequential. The "superposition principle" states that for any two vectors $|\psi\rangle,|\phi\rangle \in \mathcal{H}$ and nonzero complex numbers $\gamma, \delta$ satisfying $|\gamma|^{2}+|\delta|^{2}=1$, the linear superposition $\gamma|\psi\rangle+\delta|\phi\rangle \in \mathcal{H}$ is also a state of the system under consideration. However, unlike a global phase, the relative phase in a superposition is physically significant, i.e. $\gamma|\psi\rangle+\delta|\phi\rangle$ and $\gamma|\psi\rangle+\delta e^{i \vartheta}|\phi\rangle$ represent two different states of the same physical system. The superposition principle is fundamental to quantum theory. In fact, almost all nonclassical properties exhibited by quantum systems, e.g., nonorthogonality of quantum states [1], quantum interference [1, 2], quantum entanglement $[1,3]$, and quantum coherence [4] are consequences of quantum superpositions.

Recently a basic question, closely related to quantum superposition, was considered [5]: Does there exist a quantum operation that would superpose two unknown pure quantum states with some complex weights? The question is of particular interest because quantum theory is known to forbid physical realizations of certain operations, even plausible ones [615], and therefore, it is important to understand whether similar restrictions are also in place on something as basic as the creation of quantum superpositions. Besides, exploring such questions often reveals new ways of manipulating quantum systems that have found useful applications in quantum information and computation.

Before we proceed, we note here that a special case of the above question was initially posed in Ref. [16] where the authors asked about the existence of a quantum adder, a unitary operator that would add two unknown pure quantum states, and proved that such a unitary operator cannot exist. The proof followed from the observation that an unobservable global phase associated with the input state can distribute itself in infinitely many ways in a superposition, thereby leading to infinitely many superpositions with observable relative phases, which is unphysical.

Let us now consider the general formulation of the question on the existence of quantum superposers [5]: For given nonzero complex numbers $\alpha, \beta$ satisfying $|\alpha|^{2}+|\beta|^{2}=1$ and any given pair of vectors $|\psi\rangle,|\phi\rangle \in \mathcal{H}$, does there exist a quantum protocol that prepares the superposed state $|\Psi\rangle \propto \alpha|\psi\rangle+\beta|\phi\rangle$ ? The ambiguity of the relative phase, which ruled out the existence of a quantum adder, however, is also present in this general formulation. Let $\rho_{\chi}=|\chi\rangle\langle\chi|$ denote the density matrix for any normalized vector $|\chi\rangle$. Then it is easy to see that $\rho_{\Psi}$ cannot be a well-defined function of $\rho_{\psi}$ and $\rho_{\phi}$ for the simple reason that the density matrix $\rho_{\chi}$ corresponds not only to $|\chi\rangle$ but also to any other normalized vector $\left|\chi^{\prime}\right\rangle=e^{i \theta}|\chi\rangle$. The authors [5] therefore relaxed the definition of superposing such that there's no phase ambiguity. Specifically, for any pair of vectors $|\psi\rangle,|\phi\rangle \in \mathcal{H}$ they allowed for complex superpositions of any two vectors with density matrices $\rho_{\psi}$ and $\rho_{\phi}$. With this, the question becomes well defined. Then a superposition protocol, if one such exists, could be realized by application of a quantum channel on the input systems and then tracing out one of them. The authors also allowed post-selection, which entails the possibility of obtaining the desired output with some nonzero probability. In other words, the most general class of quantum operations, described in terms of trace-nonincreasing completely positive (CP) maps, was considered in Ref. [5]. The answer, however, turned out to be no.

Theorem 1. [5] Let $\alpha, \beta$ be any two nonzero complex numbers satisfying $|\alpha|^{2}+|\beta|^{2}=1$. Let $\mathcal{H}$ be a Hilbert space, where $\operatorname{dim} \mathcal{H} \geq 2$. Then there does not exist a nonzero $C P$ map $\Lambda_{\alpha, \beta}: \mathcal{H}^{\otimes 2} \rightarrow \mathcal{H}$ such that for all pure states $\rho_{\psi}, \rho_{\phi} \in$ $\mathcal{H}, \Lambda_{\alpha, \beta}\left(\rho_{\psi} \otimes \rho_{\phi}\right) \propto|\Psi\rangle\langle\Psi|$, where $|\Psi\rangle=\alpha|\psi\rangle+\beta|\phi\rangle$ and the states appearing in the superposition may in general depend on both $\rho_{\psi}$ and $\rho_{\phi}$.

Noting that the superposition in general may depend on both $\rho_{\psi}$ and $\rho_{\phi}$ and a global phase is not of any consequence, one has the following corollary.

Corollary 1. Let $\alpha, \beta$ be any two nonzero complex numbers satisfying $|\alpha|^{2}+|\beta|^{2}=1$. Let $\mathcal{H}$ be a Hilbert space, where $\operatorname{dim} \mathcal{H} \geq 2$. Then there does not exist a nonzero CP map $\Lambda_{\alpha, \beta}: \mathcal{H}^{\otimes 2} \rightarrow \mathcal{H}$ such that for all pure states $|\psi\rangle,|\phi\rangle \in \mathcal{H}$, $\Lambda_{\alpha, \beta}\left(\rho_{\psi} \otimes \rho_{\phi}\right) \propto|\Psi\rangle\langle\Psi|$, where $|\Psi\rangle=\alpha|\psi\rangle+\beta e^{i \theta}|\phi\rangle$ for some phase $\theta \in[0,2 \pi)$ that may in general depend on the input states.

The above result, known as the no-superposition theorem, 
forbids the existence of a universal probabilistic quantum superposer: a quantum operation that would superpose two unknown pure quantum states with nonzero probability. The result forms yet another no-go theorem in quantum theory.

Now the no-go theorems [6-15] in quantum theory are of particular significance because they tell us which operations are physically allowed and which are not. For example, the no-cloning theorem [6] states that it is impossible to make exact copies of an unknown quantum state. But at a more fundamental level, the no-go theorems can have deep implications. For example, in a world without the no-cloning theorem, it is possible to send signals faster than light $[17,18]$ and reliably distinguish nonorthogonal states, both of which would lead to a complete breakdown of our existing physical theories. So while the no-go theorems are fairly easy to understand, their implications can be far reaching, but often not immediate.

The implications of the no-superposition theorem, however, remain poorly understood so far. Neither do we know of any relation with any other existing no-go result, nor do we know of the consequences, if any, should it be violated. Although follow-up papers have come up with interesting results [1921] and variants [22], none could account for the most basic questions: Why is it not possible to superpose unknown quantum states, even with a nonzero probability? And what would be the consequences if we could?

In this paper, we will show that the existence of universal probabilistic quantum superposers implies the existence of protocols that can perform certain quantum state discrimination and cloning tasks forbidden not only in quantum theory, but also in no-signaling theories. So indeed, there will be unphysical consequences should such quantum superposers exist.

We begin by assuming that universal probabilistic quantum superposers exist. That is,

Assumption. For every pair of nonzero complex numbers $\alpha, \beta$ satisfying $|\alpha|^{2}+|\beta|^{2}=1$, there exists a universal probabilistic quantum superposer $\mathcal{Q}_{\alpha, \beta}$ that for any two pure quantum states $|\psi\rangle,|\phi\rangle$ prepares, with probability $p_{\psi, \phi}^{\alpha, \beta}>0$, a superposition state $|\Psi\rangle \propto \alpha|\psi\rangle+\beta e^{i \theta}|\phi\rangle$ for some phase $\theta \in[0,2 \pi)$, where $\theta$ may in general depend on the input states.

Thus $\mathcal{Q}_{\alpha, \beta}$ is a two-input, single-output, quantum black box that takes a pair of pure quantum states as input and generates their linear superposition as output with some nonzero probability which is allowed to vary over input states (for the sake of full generality).

The basic idea is to show that the existence of $\mathcal{Q}_{\alpha, \beta}$ implies violation of the following theorems:

Let $S_{\psi}=\left\{\left|\psi_{1}\right\rangle,\left|\psi_{2}\right\rangle, \ldots,\left|\psi_{n}\right\rangle\right\}$ be a set of pure states such that $0 \leq\left|\left\langle\psi_{i} \mid \psi_{j}\right\rangle\right|<1$ for $i \neq j$. Then,

- the states can be unambiguously distinguished (i.e., every state in the set can be correctly identified with a nonzero probability) if and only if they are linearly independent [23].
- the states can be probabilistically cloned if and only if they are linearly independent [24].

Note that the above two statements are equivalent in the following sense: For any given set of states, if unambiguous discrimination is possible, then probabilistic cloning is possible as well and vice versa. Further note that the constraint on probabilistic cloning of states follows from the condition of no faster-than-light signaling [18]. So quantum theory, and independently, the no-signaling condition, forbid unambiguous discrimination and probabilistic cloning of linearly dependent pure states. This in turn implies the following:

- Let $S_{\psi}=\left\{\left|\psi_{1}\right\rangle,\left|\psi_{2}\right\rangle, \ldots,\left|\psi_{n}\right\rangle\right\}$ be a set of linearly dependent pure states. Then, there does not exist a quantum protocol that achieves the state transformation $\left|\psi_{i}\right\rangle \rightarrow\left|\Psi_{i}\right\rangle$ for every $i$ with a nonzero probability such that the states $\left|\Psi_{1}\right\rangle,\left|\Psi_{2}\right\rangle, \ldots,\left|\Psi_{n}\right\rangle$ are linearly independent.

The proof is simple. Suppose that a quantum system is prepared in a state chosen from the known set $S_{\psi}$ but we do not know which state. Now as the states $\left|\psi_{i}\right\rangle$ are linearly dependent, quantum theory will not allow us to correctly identify the state of the system, or make copies of it, even with nonzero probability. But it is easy to see that both the tasks become possible if there exists a protocol that achieves the transformation $\left|\psi_{i}\right\rangle \rightarrow\left|\Psi_{i}\right\rangle$ with nonzero probability for every $i$, where the states $\left|\Psi_{1}\right\rangle,\left|\Psi_{2}\right\rangle, \ldots,\left|\Psi_{n}\right\rangle$ are linearly independent. Therefore such a protocol can not exist.

Let us now consider a $\mathcal{Q}_{\alpha, \beta}$-based state transformation protocol that works as follows. We feed our quantum machine $\mathcal{Q}_{\alpha, \beta}$ with two input states: The first is chosen from a known set $S_{\psi}=\left\{\left|\psi_{1}\right\rangle,\left|\psi_{2}\right\rangle, \ldots,\left|\psi_{n}\right\rangle\right\}$ of pure states and the second is some pure state $|\phi\rangle$. In this way it is possible to prepare an ensemble $S_{\Psi}=\left\{\left|\Psi_{1}\right\rangle,\left|\Psi_{2}\right\rangle, \ldots,\left|\Psi_{n}\right\rangle\right\}$ of output states, where each state $\left|\Psi_{j}\right\rangle \propto \alpha\left|\psi_{j}\right\rangle+\beta e^{i \theta_{j}}|\phi\rangle$ is generated with some nonzero probability $p_{\psi_{i}, \phi}^{\alpha, \beta}$. Thus we have a protocol that transforms $\left|\psi_{j}\right\rangle \rightarrow\left|\Psi_{j}\right\rangle$ with a nonzero probability for every $j$.

For a given $\mathcal{Q}_{\alpha, \beta}$ and $S_{\psi}$, observe that the output states will be different for different choices of $|\phi\rangle$. To make this explicit, denote the set of output states by $S_{\Psi}(\phi)$. So if the states $\left|\psi_{1}\right\rangle,\left|\psi_{2}\right\rangle, \ldots,\left|\psi_{n}\right\rangle$ are linearly dependent, then for every $|\phi\rangle$ the states in $S_{\Psi}(\phi)$ must also be linearly dependent because otherwise, the protocol would be unphysical.

We now give a simple proof that the protocol, in fact, is unphysical for all $\mathcal{Q}_{\alpha, \beta}$. Consider a set of three linearly dependent pure states that belong to a $d$-dimensional Hilbert space, where $d \geq 3$. The states are given by

$$
\begin{aligned}
& \left|\psi_{1}\right\rangle=|\psi\rangle, \\
& \left|\psi_{2}\right\rangle=\left|\psi^{\perp}\right\rangle, \\
& \left|\psi_{3}\right\rangle=a|\psi\rangle+b\left|\psi^{\perp}\right\rangle,
\end{aligned}
$$

where $a, b \neq 0, a, b \in \mathbb{R}, a^{2}+b^{2}=1$. By construction, the states are linearly dependent as they live in the twodimensional subspace spanned by $\left\{|\psi\rangle,\left|\psi^{\perp}\right\rangle\right\}$. 
Following the protocol, we feed $\mathcal{Q}_{\alpha, \beta}$ with two input states, where the first input is chosen from $\left\{\left|\psi_{1}\right\rangle,\left|\psi_{2}\right\rangle,\left|\psi_{3}\right\rangle\right\}$ as given above, and the second input state is taken to be a pure state $|\phi\rangle$ which is orthogonal to both $|\psi\rangle$ and $\left|\psi^{\perp}\right\rangle$. Then the possible output states, each of which is generated with some nonzero probability, are given by

$$
\begin{aligned}
& \left|\Psi_{1}\right\rangle=\alpha\left|\psi_{1}\right\rangle+\beta e^{i \theta_{1}}|\phi\rangle, \\
& \left|\Psi_{2}\right\rangle=\alpha\left|\psi_{2}\right\rangle+\beta e^{i \theta_{2}}|\phi\rangle, \\
& \left|\Psi_{3}\right\rangle=\alpha\left|\psi_{3}\right\rangle+\beta e^{i \theta_{3}}|\phi\rangle .
\end{aligned}
$$

We will show that the states $\left|\Psi_{j}\right\rangle$ for $j=1,2,3$ are linearly independent; that is, the equation

$$
x_{1}\left|\Psi_{1}\right\rangle+x_{2}\left|\Psi_{2}\right\rangle+x_{3}\left|\Psi_{3}\right\rangle=0
$$

holds if and only if $x_{1}=x_{2}=x_{3}=0$. The if part is trivial. So let us now consider the only if part.

First, we write Eq. (3) as

$\alpha\left(x_{1}+a x_{3}\right)|\psi\rangle+\alpha\left(x_{2}+b x_{3}\right)\left|\psi^{\perp}\right\rangle+\beta \sum_{j=1}^{3} e^{i \theta_{j}} x_{j}|\phi\rangle=0$

As the states $|\psi\rangle,\left|\psi^{\perp}\right\rangle,|\phi\rangle$ are mutually orthogonal they are linearly independent. Thus the coefficients appearing in the above superposition must vanish, i.e.,

$$
\begin{aligned}
\alpha\left(x_{1}+a x_{3}\right) & =0, \\
\alpha\left(x_{2}+b x_{3}\right) & =0, \\
\beta\left(e^{i \theta_{1}} x_{1}+e^{i \theta_{2}} x_{2}+e^{i \theta_{3}} x_{3}\right) & =0 .
\end{aligned}
$$

Since $\alpha, \beta \neq 0$, we have

$$
\begin{aligned}
x_{1}+a x_{3} & =0, \\
x_{2}+b x_{3} & =0, \\
e^{i \theta_{1}} x_{1}+e^{i \theta_{2}} x_{2}+e^{i \theta_{3}} x_{3} & =0 .
\end{aligned}
$$

Let us now find the conditions under which the above three equations are satisfied simultaneously. As $a, b \neq 0$, we see that the above three equations are simultaneously satisfied when $x_{i}=0$ for all $i=1,2,3$. We will now show that this is the only solution. To establish this, assume that $x_{i} \neq 0$ for all $i=1,2,3$. Then from (8) and (9) we get $x_{1}=-a x_{3}$, and $x_{2}=-b x_{3}$. Substituting these in (10) and noting that $x_{3} \neq 0$ we obtain

$$
e^{i \theta_{1}} a+e^{i \theta_{2}} b-e^{i \theta_{3}}=0,
$$

or, equivalently,

$$
a+e^{i \theta_{21}} b=e^{i \theta_{31}},
$$

where $\theta_{21}=\theta_{2}-\theta_{1}$ and $\theta_{31}=\theta_{3}-\theta_{1}$. Equation (12) implies that

$$
\left|a+b^{\prime}\right|=1,
$$

where $b^{\prime}=e^{i \theta_{21}} b$. Now, recall that $a, b \neq 0, a, b \in \mathbb{R}$, and $a^{2}+b^{2}=1$. Then

$$
a^{2}+\left|b^{\prime}\right|^{2}=1
$$

A simple calculation shows that Eqns. (13) and (14) are satisfied only when $\theta_{21}=\pi / 2,3 \pi / 2$ (since, $a, b \neq 0$ ). Then from (12) it follows that $a=\cos \theta_{31}$ and $b= \pm \sin \theta_{31}$. But the phases that $\mathcal{Q}_{\alpha, \beta}$ associate with the superposition cannot have any dependence on $a$ and $b$ ! This is because $a$ and $b$ are basis-dependent coefficients and $\left|\psi_{3}\right\rangle$ has infinitely many such representations. In other words, while $\theta_{3}$ may depend on $\left|\psi_{3}\right\rangle$ and $|\phi\rangle$, it cannot depend on the basis representation of $\left|\psi_{3}\right\rangle$. So the solutions are not feasible.

Therefore, Eqns. (8), (9), and (10) can only be simultaneously satisfied when $x_{i}=0, i=1,2,3$. Consequently, the only solution to (3) is $x_{1}=x_{2}=x_{3}=0$. Thus the states $\left|\Psi_{1}\right\rangle,\left|\Psi_{2}\right\rangle,\left|\Psi_{3}\right\rangle$ are linearly independent. Note that the analysis holds for all $\mathcal{Q}_{\alpha, \beta}$. This completes the proof.

Thus we have shown that the existence of $\mathcal{Q}_{\alpha, \beta}$ implies the existence of a protocol that can transform linearly dependent pure states into linearly independent pure states. As explained earlier, such protocols can be used to perform the tasks of unambiguous discrimination and probabilistic cloning of linearly dependent pure states, both of which are forbidden in quantum theory and also violate the no-signaling condition. So the unconditional superposition of unknown quantum states, even probabilistically, gives rise to unphysical consequences.

Now, interestingly, there exists a probabilistic quantum protocol to superpose two unknown pure states [5]. Not surprisingly though, the protocol comes with strings attached - in particular, each input state must have a fixed overlap with a known reference state and the superposition coefficients are functions of the overlaps as well. It is now clear why these conditions are necessary, for without these constraints probabilistic superposition would not be possible.

Our result also sheds light on a recent theorem [22] which states that it is possible to superpose two unknown pure states chosen from a known set if and only if the states are linearly independent. The if part here is easy to understand: If the states are linearly independent, then in the first step, one correctly identifies the input states by performing suitable unambiguous state discrimination measurements and in the next step creates the desired superposition. The only if part, on the other hand, can be understood by noting that $Q_{\alpha, \beta}$ can transform linearly dependent pure states into linearly independent ones, which is unphysical. This implies the states in the given set must all be linearly independent.

Conclusions. The no-go theorems in quantum theory help us to understand the class of allowed physical operations. But it is also equally important to understand the consequences should no-go theorems be violated and the answers must come from physics. Here we showed that the existence of a protocol that superposes unknown pure states, even with nonzero probability, leads to unambiguous discrimination and probabilistic cloning of linearly dependent pure states - tasks that are forbidden in quantum theory and also in no-signaling theories.

One question, in the context of the present result, however, remains open: What kind of unphysical consequences would arise if a universal probabilistic quantum superpower, assum- 
ing it exists, is allowed to admit only qubit states? We could not find a satisfactory answer. Nevertheless, we are hopeful that a satisfactory answer will eventually be found, perhaps considering a different physical scenario.

The author is grateful to Guruprasad Kar and Tomasz Paterek for many helpful discussions.

*som@jcbose.ac.in; som.s.bandyopadhyay@gmail.com

[1] Any standard textbook of quantum mechanics (for example, The Feynman Lectures on Physics, Vol. 3).

[2] A. Zeilinger, Experiment and the foundations of quantum physics, Rev. Mod. Phys. 71, S288 (1999).

[3] R. Horodecki, P. Horodecki, M. Horodecki, and K. Horodecki, Quantum entanglement, Rev. Mod. Phys. 81, 865 (2009).

[4] A. Streltsov, G. Adesso, and M. B. Plenio, Colloquium: Quantum coherence as a resource, Rev. Mod. Phys. 89, 041003 (2017).

[5] M. Oszmaniec, A. Grudka, M. Horodecki, and A. Wójcik, Creating a Superposition of Unknown Quantum States, Phys. Rev. Lett. 116, 110403 (2016).

[6] W. K. Wootters and W. H. Zurek, A single quantum cannot be cloned, Nature (London) 299, 802 (1982).

[7] D. Dieks, Communication by EPR devices,Phys. Lett. A 92, 271 (1982).

[8] H. Barnum, C. M. Caves, C. A. Fuchs, R. Jozsa, and B. Schumacher, Noncommuting Mixed States Cannot Be Broadcast, Phys. Rev. Lett. 76, 2818 (1996).

[9] Daniel R. Terno, Nonlinear operations in quantum-information theory, Phys. Rev. A 59, 3320

[10] A. K. Pati and S. L. Braunstein, Impossibility of deleting an unknown quantum state, Nature (London) 404, 164 (2000).

[11] A. K. Pati, General impossible operations in quantum informa- tion, Phys. Rev. A 66, 062319 (2002).

[12] M. Piani, P. Horodecki, and R. Horodecki, No-LocalBroadcasting Theorem for Multipartite Quantum Correlations, Phys. Rev. Lett. 100, 090502 (2008).

[13] M. Lostaglio and M. P. Müller, Coherence and Asymmetry Cannot be Broadcast, Phys. Rev. Lett. 123, 020403 (2019).

[14] M. Araújo, A. Feix, F. Costa, and Č. Brukner, Quantum circuits cannot control unknown operations, New J. Phys. 16093026 (2014).

[15] J. Thompson, K. Modi, V. Vedral, and M. Gu, Quantum plug n' play: modular computation in the quantum regime, New J. Phys. 20013004 (2018).

[16] U. Alvarez-Rodriguez, M. Sanz, L. Lamata, and E. Solano, The forbidden quantum adder, Sc. Rep. 5, 11983 (2015).

[17] N. Gisin, Quantum cloning without signaling, Phys. Lett. A 242, 1 (1998).

[18] L. Hardy and D. D. Song, No signalling and probabilistic quantum cloning, Phys. Lett. A 259, 331 (1999).

[19] S. Dogra, G. Thomas, S. Ghosh, and D. Suter, Superposing pure quantum states with partial prior information, Phys. Rev. A 97, 052330 (2018).

[20] M. Doosti, F. Kianvash, and V. Karimipour, Universal superposition of orthogonal states, Phys. Rev. A 96, 052318 (2017).

[21] S. Sami and I. Chakrabarty, A note on superposition of two unknown states using Deutsch CTC model, Mod. Phys. Lett. A 31, 1650170 (2016).

[22] M.-X. Luo, H.-R. Li, H. Lai, and X. Wang, Unified quantum no-go theorems and transforming of quantum pure states in a restricted set, Quantum Inf. Process. 16, 297 (2017).

[23] A. Chefles, Unambiguous discrimination between linearly independent quantum states, Phys. Lett. A 239, 339 (1998).

[24] L.-M. Duan and G.-C. Guo, Probabilistic Cloning and Identification of Linearly Independent Quantum States, Phys. Rev. Lett. 80, 4999 (1998). 